\title{
La lettre dans la bergerie. Épistolaire pastoral et fiction d'une rhétorique naturelle à la fin de la Renaissance (1571-1632)
}

\section{Suzanne Duval}

\section{(2) OpenEdition}

Journals

Édition électronique

URL : http://journals.openedition.org/rhetorique/790

DOI : $10.4000 /$ rhetorique. 790

ISSN : 2270-6909

Éditeur

UGA Éditions/Université Grenoble Alpes

Édition imprimée

ISBN : 978-2-37747-066-2

Référence électronique

Suzanne Duval, « La lettre dans la bergerie. Épistolaire pastoral et fiction d'une rhétorique naturelle à la fin de la Renaissance (1571-1632) », Exercices de rhétorique [En ligne], 12 | 2019, mis en ligne le 28 janvier 2019, consulté le 12 septembre 2020. URL : http://journals.openedition.org/rhetorique/790 ; DOI : https://doi.org/10.4000/rhetorique.790

Ce document a été généré automatiquement le 12 septembre 2020.

\section{c) (1)(2)}

Les contenus de la revue Exercices de rhétorique sont mis à disposition selon les termes de la Licence Creative Commons Attribution - Pas d'Utilisation Commerciale - Partage dans les Mêmes Conditions 4.0 International. 


\title{
La lettre dans la bergerie. Épistolaire pastoral et fiction d'une rhétorique naturelle à la fin de la Renaissance (1571-1632)
}

\author{
Suzanne Duval
}

1 Décrivant le genre des " histoires de bergers ", Charles Sorel observe que " ce qu'on y trouve en general a peu de vray-semblable ${ }^{1} »$. L'hétérogénéité textuelle de la fiction pastorale est, selon le même auteur, la principale source de ce défaut :

ces personnages rustiques font leurs recherches avec les mesmes artifices que les Courtisans les plus polis; Ils escrivent des Lettres fort eloquentes et fort tendres ; Ils composent des Vers merveilleux \& les chantent si bien qu'ils ravissent ceux qui les écoutent ${ }^{2}[. .$.$] .$

La lettre mondaine a pourtant un tropisme particulier pour la littérature pastorale, comme l'attestent, dans les recueils épistolaires du premier XVII ${ }^{\mathrm{e}}$ siècle, les Diane et les Thyrcis fictifs ou à clefs à qui s'adressent les orateurs mondains. L'imaginaire langagier d'un style épistolaire naturel et spontané s'inscrit en effet harmonieusement dans l'allégorie arcadienne d'un loisir académique et mondain naturalisé3. Il m'a donc paru intéressant d'étudier la place de la rhétorique de l'épître dans l'économie des bergeries en prose de la fin de la Renaissance, sachant que la lettre d'amour deviendra à l'époque galante, selon la formule d'Adrienne Petit, le «laboratoire d'une nouvelle rhétorique des passions ${ }^{4} »$. 


\section{La lettre enchâssée : geste anthologique et acculturation rhétorique}

\section{La lettre fictionnelle au carrefour des essors éditoriaux}

3 La variété rhétorique est un trait constitutif de la poétique du roman baroque ${ }^{5}$ et à plus forte raison de la veine des fictions pastorales, particulièrement vivace entre les années 1560 et 1620 , dans un contexte d'essor éditorial pour les recueils de pièces en prose et en vers. Le succès, à l'automne de la Renaissance, du Trésor des Amadis, témoigne du goût prononcé du lectorat pour la disposition anthologique, ainsi que pour la vertu exemplaire et modélisatrice de l'ornement rhétorique $^{6}$ : détaché de l'intrigue chevaleresque, le recueil de pièces insérées prétend acculturer son public à la fine fleur de l'éloquence mondaine, au même titre que les divers livres de Marguerites qui sont édités et réédités à la même époque ${ }^{7}$. La fiction pastorale se pare donc elle aussi des traits caractéristiques d'un genre alors en plein essor, par sa poétique diversifiée ainsi que par son habillage éditorial : son hétérogénéité textuelle est parfois affichée dès son titre $^{8}$, et elle arbore une disposition typographique qui, par les alinéas, les intertitres, les italiques, les tables de pièces insérées et les index de lieux communs, se rapprochent de celle du recueil9.

4 La lettre enchâssée joue un rôle important dans ce processus de diversification et de valorisation du texte fictionnel pastoral, à une époque où le recueil épistolaire, sur le point de devenir le principal tremplin éditorial des écrivains mondains ${ }^{10}$, s'émancipe du manuel d'épistolographie pour constituer un genre littéraire à part entière. L'affinité de la fiction en prose avec la rhétorique épistolaire s'inscrit dans le temps long de la Renaissance et la redécouverte, au XIV ${ }^{\mathrm{e}}$ siècle, des épîtres familières de Cicéron ${ }^{11}$. Le modèle de style naturel que ces dernières offrent aux écrivains, associé à la référence poétique des Héroïdes d'Ovide, est au fondement de l'immense fortune de la lettre amoureuse fictionnelle. La veine italianisante $d u$ recueil de lettres amoureuses, marquée par les prestigieux modèles de Bembo et de Piccolomini, est autorisée en France par le succès du Recueil des rymes et des proses d'Étienne Pasquier, au sein duquel on trouve une longue section épistolaire ${ }^{12}$. Cette mode sera continuée au XVII ${ }^{\mathrm{e}}$ siècle par celle des recueils d'Héroïdes, ouvrages composés de libres adaptations, le plus souvent en prose, d'Ovide ${ }^{13}$. Les anthologies mondaines, soucieuses de faire valoir leur littérarité et de se distinguer du manuel de rhétorique scolaire, ménagent elles aussi une place de choix aux lettres, en insérant entre leurs compliments classés par lieux communs de brèves suites d'épitres amoureuses qui forment des fragments de fictions par lettres ${ }^{14}$.

$5 \quad$ Insérer des épîtres dans une fiction pastorale à la fin de la Renaissance revient donc à capter une tradition poétique prestigieuse tout en répondant au goût de l'époque pour le manuel de rhétorique en forme d'anthologie. Ce geste anthologique contribue également à faire de la littérature romanesque un modèle d'éloquence ambigu et critiqué, dans la mesure où la rhétorique de la lettre amoureuse, en dépit ou à cause de son succès mondain, est un type épistolaire faiblement théorisé et peu considéré par le discours épistolographique de l'époque. 


\section{L'épistolographie de la lettre d'amour : une rubrique vide?}

6 L'engouement des genres littéraires mondains pour la lettre d'amour a partie liée au fait que les traités de rhétorique plus doctes se méfient, pour leur part, de la moralité douteuse de ce genre d'écrire, et n'en traitent que rapidement dans leurs prescriptions. D'une manière assez paradoxale, l'épitre amoureuse passe donc, en pratique, pour un bel endroit et en théorie pour un type d'écriture essentiellement affectif, ne pouvant se réduire aux éléments de la rhétorique et par conséquent plus facile à exemplifier qu'à décrire. Dans son traité épistolaire, Érasme n'en donne qu'un exposé de quelques pages inséré dans la section consacrée aux lettres délibératives. La rubrique, intitulée " amatoriae epistolae ", rassemble les préceptes sur les lettres d'amour et d'amitié, mais seule la seconde espèce est exemplifiée par une épître familière de Cicéron ${ }^{15}$. Pierre Fabri exprime quant à lui sa réticence à traiter d'un genre aussi licencieux, qu'il rattache aussitôt aux modèles d'Ovide et de Properce ${ }^{16}$. Il opère ensuite une distinction entre les lettres d'« amour vertueuse », c'est-à-dire amicales, et les lettres «d'amour vitieuse $^{17} »$ :

Quand on veult rescripre d'amour vitieuse a quelque belle jeune fille pour luy desclarer son amour-combien que j'aye esté délibéré de ne bailler point de exemple de cecy ne de vitupere, ne de invective, etc., veu que la malice des hommes en sçayt trop, touteffoys, pour aggreement et assouvissement de mon livre et par conseil je l'ay fait ${ }^{18}$.

7 La typologie que donne Fabri de la lettre d'amour vicieuse suit un ordonnancement semblable à celui des autres genres épistolaires, puisque le rhétoricien opère un classement en fonction de la visée pragmatique du scripteur ${ }^{19}$ :

-s'attirer la bienveillance du destinataire

-déclarer son amour

-offrir ses services ${ }^{20}$.

Cependant ces trois cas de figures sont loin de recouvrir les actes de langage variés que l'on rencontre dans les épitres fictionnelles, en particulier ceux de la plainte et des reproches.

9 Le Secrétaire à la mode de Jean Puget de La Serre, manuel d'épistolographie à succès que l'auteur augmente en 1651 d'une Instruction à écrire des lettres, ne consacre aucune rubrique proprement didactique à la lettre d'amour, alors même que les exemples de compliments qui sont donnés dans la suite de l'ouvrage s'inscrivent de manière explicite pour une grande partie d'entre eux dans le cadre d'une communication amoureuse $^{21}$. Un tel déficit du métadiscours rhétorique sur la lettre d'amour, qui, à l'ère de la littérature galante et classique, sera justifié par des auteurs comme Madeleine de Scudéry ou Du Plaisir qui insisteront sur la spontanéité passionnée de l'écriture épistolaire amoureuse et par conséquent la difficulté à en prescrire les règles ${ }^{22}$, marque une affinité supplémentaire entre ce genre d'écrire et celui de la fiction en prose. L'un et l'autre sont caractérisés à l'époque baroque par le succès qu'ils rencontrent auprès du public mondain et par leur présence encore peu légitime dans le champ des BellesLettres. Ce parallèle entre les deux genres en amène un autre : de la même manière que la faible légitimité théorique du genre romanesque se traduit par une surenchère ornementale et une tendance à se parer de tous les genres du bien-dire, la lettre 
enchâssée offre elle aussi un bouquet, propice au découpage anthologique, de toutes les fleurs de la langue amoureuse.

\section{La disposition des épîtres : une naturalisation du langage épistolaire}

\section{Un ornement rare et précieux du texte pastoral}

Comme les plaintes ou les vers enchâssés, la prose épistolaire amplifie la matière amoureuse de la narration fictionnelle au moyen des procédés rhétoriques qui lui sont propres, assurant ainsi variété et abondance stylistiques. La lettre se distingue cependant des autres pièces insérées par sa rareté relative. Le tableau qui suit rend compte de leur petit nombre dans l'économie générale du texte pastoral :

\begin{tabular}{|l|l|l|l|}
\hline & $\begin{array}{l}\text { Nombre de } \\
\text { pages }\end{array}$ & $\begin{array}{l}\text { Nombre de poèmes } \\
\text { insérés }\end{array}$ & $\begin{array}{l}\text { Nombre de } \\
\text { lettres } \\
\text { insérées }\end{array}$ \\
\hline Pyrenée, Belleforest (1571) & 244 & 57 & 0 \\
\hline Diane de Montemayor, trad. Colin (1578) & 209 & 36 & 5 \\
\hline Bergeries de Juliette, Montreux (1585) & 582 & 202 & 4 \\
\hline Mariane du Filomène (1591) & 244 & 5 & 3 \\
\hline $\begin{array}{l}\text { Première et deuxième partie des Aventures de } \\
\text { Floride, épisode pastoral des livres III et I, } \\
\text { Béroalde de Verville (1601) }\end{array}$ & 506 & $\begin{array}{l}\text { regroupés à la fin } \\
\text { du livre }\end{array}$ & 5 \\
\hline Fantaisies amoureuses (1601) & 479 & 30 & 43 \\
\hline Paradis d'amour, Vauquelin de La Frenaye (1606) & 120 & 44 & 39 \\
\hline Première partie de L'Astrée, Urfé (1612) & 408 & 45 & 26 \\
\hline Deuxième partie de L'Astrée, Urfé (1614) & 431 & 38 & 3 \\
\hline Diane Françoise, Du Verdier (1624) & 804 & 8 & 3 \\
\hline 6 premiers livres du Berger extravagant (1627) & 961 & 3 & 4 \\
\hline Diane des bois, Préfontaine (1632) & 285 & 39 \\
\hline
\end{tabular}

11 Le nombre de lettres, relativement faible quelle que soit l'ampleur du livre, est inférieur à celui des poèmes insérés (et il serait intéressant, dans cette perspective, de comparer leur présence quantitative à celle des plaintes en prose). Honoré d'Urfé se démarque des autres auteurs par une présence particulièrement importante de l'épistolaire dans son œuvre. D'un point de vue diachronique, l'évolution montre que la 
lettre, éventuellement absente dans les textes de la fin de la Renaissance (comme dans La Pyrenée), devient un ornement obligé au XVII ${ }^{\mathrm{e}}$ siècle, dont la présence se maintient alors même que le nombre de poèmes insérés se réduit et peut parfois devenir inférieur à celui des épîtres (chez Du Verdier et Préfontaine). Il reste qu'au regard de la prose du récit, la lettre insérée est dans l'ensemble du corpus nettement minoritaire: elle apparait donc comme un ornement rare, mais rendu remarquable par sa typographie et sa mise en scène narrative.

\section{La scène de réception épistolaire comme rhétorique d'une lecture sensible}

La disposition de l'épître au sein du récit suit en effet une logique d'intensification du texte fictionnel. Son énonciation à la première personne et au présent constitue déjà une modulation expressive de l'énoncé narratif. La lettre apparaît comme la pièce centrale d'une séquence narrative dramatisée, marquant l'acmé des passages obligés de l'intrigue amoureuse que sont la déclaration d'amour, la séparation des amants ou encore leur brouille passagère ${ }^{23}$. Les lieux communs épistolaires pragmatiquement orientés que sont les propositions de service ${ }^{24}$, les reproches ${ }^{25}$, les excuses ${ }^{26}$ ou encore la plainte ${ }^{27}$ fonctionnent de manière indissociable comme le catalyseur d'un point nodal de l'action et comme un enrichissement rhétorique de la matière amoureuse. Il est remarquable, à ce titre, que la narration de la transmission de la lettre mette plutôt l'accent sur la réception du message que sur sa production. Certes, Charles Sorel, dans le Berger extravagant, fait exception à la règle en détaillant le long moment que prend Lysis pour rédiger une lettre de compliments à Charite, veillant " toute la nuict " pour " escrire et effacer mille resveries ${ }^{28}$ ". Cette variation du topos tient à l'intention satirique de l'auteur qui, en attirant l'attention sur l'écriture du texte épistolaire, invite son public à percevoir les rouages artificiels des différentes pièces de l'énoncé fictionnel et à ne pas en être dupe. Inversement, l'ellipse des conditions d'écriture de la lettre dans la fiction pastorale alimente le mythe d'une rhétorique épistolaire naturelle, aussi spontanée que celle de la conversation. Et, réciproquement, les figures pathétiques qui amplifient la scène de réception épistolaire pastorale fonctionnent comme une rhétorique de la lecture naturalisée. Elles signalent le lieu textuel de la lettre comme celui d'une communion sensible où toute distance entre l'énoncé fictionnel et le lecteur s'abolit. Aussi est-il fréquent que le narrateur intervienne immédiatement à la suite de la citation de l'épître au moyen d'une figure de communication adressée à son public ${ }^{29}$, pour représenter l'effet que la lecture de la lettre produit sur le personnage intéressé, comme dans cet exemple tiré des Bergeries de Juliette de Montreux :

Tant s'en faut amoureuse bande, que Diane print quelque goust en ces lettres, ou qu'elle y mist son entendement, qu'elle ne sçavoit qu'elle lisoit, tant elle estoit transportée de l'amour du Felicio ${ }^{30}[. .$.$] .$

Le lecteur est invité, par le biais de l'apostrophe, à prendre part au «transport » de la bergère. L'efficacité du discours épistolaire réside dans cette capacité à passer outre les multiples médiations dont il est le produit : la distance temporelle et spatiale qui sépare les acteurs de la communication, le montage fictionnel qui préside à l'enchâssement de la lettre sont comme annulés par la sympathie universelle que celle-ci suscite et qui rassemble dans un partage sensible narrateur, personnages et lecteurs. Cette communion peut également prendre la forme d'une exclamation de la voix narrative, 
comme dans ce passage de L'Astrée où la bergère éponyme retrouve une lettre de Céladon et s'aperçoit, à sa lecture, combien elle a été injuste envers son amant :

o quels cousteaux tranchans furent ces paroles en son ame, lorsqu'elles luy remirent en mémoire le commandement qu'elle luy avoit fait, \& la resolution qu'ils avoient prise de cacher par ceste dissimulation leur amitié ${ }^{31}$ !

Support expressif par excellence, la lettre a le pouvoir de se transmuer en une présence affective et presque physique, entraînant comportements fétichistes et symptômes pathologiques de la part des destinataires. En témoigne, dans la Diane des bois de Préfontaine, la pâmoison d'Agathine à la lecture de la lettre de Thircis :

Agathine recognoissant encor à l'ouverture de ceste lettre les traicts d'une main qu'elle avoit si souvent et si passionnément baisée, devint plus bléme que la mort, tout son sang \& ses esprits s'estant retirez au cœur, les jambes luy faillirent, \& elle tomba par terre, comme un corps privé de vie. Apres qu'elle fut revenuë à elle, elle considere attentivement tous ces traicts ; \& chaque syllabe tire un million de triste souspirs du fond de sa poictrine embrasée de l'amour de son Berger, \& brisée de regrets de l'avoir si laschement offensés ${ }^{32}$.

15 Les «traicts» manuscrits forment un portrait graphique immédiatement reconnaissable de l'épistolier. Bien plus que les poèmes ou que les plaintes, les lettres passent pour le medium d'un style personnel où transparaît la singularité d'un locuteur. Astrée insiste, notamment, sur l'impossibilité d'attribuer une épître (au style pourtant particulièrement conventionnel) à un autre que Céladon :

Ah ma sœur ! interrompit Astrée, que c'est bien Celadon qui a escrit ces paroles! je

le reconnois à la façon d'escrire \& de parler ${ }^{33}[. .$.$] .$

La mise en scène de la réception sensible de la lettre naturalise le procédé de l'enchâssement épistolaire en le fondant dans une narration pathétique, et présente au lecteur un modèle de lecture essentiellement affectif. L'épître apparaît ainsi comme une pièce de rhétorique typiquement mondaine, qui mobilise les facultés du cœur bien plus que celles de l'entendement. L'art du bien-dire qui la sous-tend passe lui aussi pour une forme d'acculturation rhétorique spontanée, contenant en quelques périodes tout l'art du parler roman.

\section{Le parler roman mis en pièces}

\section{Brièveté et agencements périodiques du texte épistolaire enchâssé}

17 Contrairement aux plaintes et aux poèmes strophiques qui bien souvent courent sur plusieurs feuillets, l'épître dépasse rarement une longueur de deux pages, ce qui facilite son repérage et sa lecture rapides. Une telle brièveté correspond aux prescriptions données par les manuels d'épistolographie ${ }^{34}$. Selon Charles Sorel, le style épistolaire, quel que soit le type de la lettre, obéit aux règles traditionnelles de la disposition rhétorique tout en étant fortement contraint par la nécessité pragmatique de ne pas être «importun ${ }^{35}$ ». L'épistolier doit donc, dans la mesure du possible, contenir l'ensemble de son discours « dans les bornes d'une periode, ou de deux, ou de trois, \& l'on les peut arranger sans beaucoup d'artifice ${ }^{36} »$.

18 Les lettres insérées, conformément à cette règle de brièveté, font souvent l'économie de la formule d'appel et annoncent ex abrupto le sujet de la lettre, parfois introduit par le biais d'un énoncé sentencieux. Elles se composent d'un petit nombre de périodes dont l'agencement est soigné. En contexte fictionnel, ce trait est particulièrement saillant 
dans la mesure où la prose narrative de l'époque baroque se caractérise au contraire par une syntaxe paratactique rarement interrompue par des points, qui tend à dissoudre le moule périodique dans un flux continu. Le contraste est alors d'autant plus sensible que l'exorde de la lettre, afin de capter l'intérêt du lecteur, prend généralement la forme d'une brève période aphorisable, qui fonctionne comme la majeure universelle du raisonnement effectué dans la suite du propos ${ }^{37}$. En voici un exemple tiré de la première partie de L'Astrée d'Honoré d'Urfé :

Quant à moy ce fut par mesgarde, il peut bien estre que Daphnis le fit à dessein, \& nous trouvant si pres de luy, je fus contrainte de le considerer : auparavant il estoit assis, \& appuyé contre un arbre : mais à ce coup nous le trouvasmes couché de son long en terre un bras sous la teste, \& sembloit qu'il veillast : car il avoit devant luy une lettre, toute moüillé des pleurs qui luy couloient le long du visage ; mais en effet il dormoit : y ayant apparence, que lisant ce papier le travail du chemin avec ses profonds pensers l'eust peu à peu assoupy: nous en fusmes encores plus certaines, quand Daphnis plus asseurée que moy, se baissant lentement, m'apporta la lettre toute moüillée des larmes qui trouvoient passage sous sa paupiere mal close, cette veuë me toucha de pitié : mais beaucoup plus sa lettre qui estoit telle. LETTRE DE FILANDRE A DIANE

Ceux qui ont l'honneur de vous voir courent une dangereuse fortune ${ }^{38}$.

La période narrative progresse par accumulations de circonstances intégrées au moyen de conjonctions de coordination et de participes passés et présents. L'exorde de la lettre, au contraire, abrège ostensiblement l'énoncé en commençant ex abrupto, sans appel du destinataire, par une considération générale qui arbore les traits caractéristiques du style sentencieux. Une seconde période, plus amplifiée, introduit la thèse du discours au moyen d'une figure de dilemme :

S'ils vous aiment ils sont outrecuidez, \& s'ils ne vous aiment point, ils sont sans jugement; vos perfections estant telles, qu'avec raison elles ne peuvent, ny estre aimées ny n'estre point aimées : \& moy estant contraint de tomber en l'une de ces deux erreurs, j'ay choisi celle qui a plus esté selon mon humeur, \& dont aussi bien il m'estoit impossible de me retirer ${ }^{39}$.

20 Le moule périodique se scinde en deux parties, la protase consistant dans la formulation générale du dilemme («S'ils vous aiment [...] point aimées»), et l'apodose dans l'application du raisonnement au cas particulier de l'épistolier. L'architecture périodique est soulignée par l'organisation harmonieuse des isotopies (passion/nécessité /nécessité/passion/), les parallélismes ("s'ils vous aiment/ne vous ayment point », " ny estre aimées/ny n'estre point aymées »). Le propos en vient ensuite, au moyen de deux périodes beaucoup plus courtes, à la réfutation :

Ne trouvez donc mauvais, belle Diane, puis qu'on ne vous peut voir sans vous aimer, que vous ayant veuë je vous aime. Que si cette temerité merite chastiement, ressouvenez-vous que j'aime mieux vous aimer en mourant, que vivre sans vous aimer ${ }^{40}$.

21 La brièveté des phrases marque la visée interlocutive de cette partie du discours, de même que l'adresse à la destinataire. Quoique d'allure plus conversationnelle et en apparence plus naturelle, l'harmonie périodique arbore les artifices traditionnels des figures gorgianiques (allitération en [v], parallélisme « voir sans vous aimer/vous ayant veuë je vous aime ", antithèse "vous aimer en mourant/vivre sans vous aimer »). Une figure de correction, caractéristique de cet effet de style naturel, permet enfin d'introduire la péroraison, formée de la plus ample période de la lettre, et d'en atténuer la véhémence rhétorique. Comme c'était le cas pour l'exorde, la reprise de l'énoncé narratif aussitôt après accuse un fort contraste syntaxique et rythmique : 
Mais, que dis-je, j'aime mieux? il n'est plus en mon choix: car il faut que par nécessité je sois tant que je vivray, aussi veritablement vostre serviteur, que vous ne sçauriez estre telle que vous estes, sans estre la plus belle Bergere qui vive.

A peine pûs-je achever cette lettre que je m'en retournay toute tremblante, \& Daphnis la remit si doucement où elle l'avoit prise, qu'il ne s'en esveilla point, \& s'en revenant à moy qui l'attendois assez pres de-là : Me permettez vous de parler? me dit-elle ${ }^{41}$. la métaphore, marques insignes, comme l'a montré Camille Esmein, du parler roman dans l'imaginaire du XVII siècle $^{42}$. Le statut de discours adressé de la lettre favorise, cependant, un goût pour la pointe spirituelle également caractéristique des anthologies de bien-dire, et beaucoup moins représenté dans les autres formes de prose fictionnelle que sont la plainte ou la narration. Le seuil et la clôture de l'épître sont ainsi des lieux signalés par la présence de lieux communs immédiatement reconnus par le lecteur accoutumé aux fleurs du bien-dire, tandis que le corps de la lettre se condense autour de quelques expressions topiques et brillantes qui énoncent le thème principal du message. Il convient pourtant de nuancer cette observation générale, puisque les bergères, autrices particulièrement bien représentées dans la littérature épistolaire pastorale, n'arborent guère ces brillants rhétoriques, qui sont plutôt l'apanage de l'éloquence masculine ${ }^{43}$.

La présence textuelle de la destinataire offre une première marque distinctive de ce parler roman par lettres, à travers des salutations et des formules d'adresse épidictiques telles que les superlatifs hyperboliques, les épithètes substantivées ou les métonymies :

Ma belle, mon cœur, et mon esprit, je cognoy maintenant que vous estes à votre aise en me voyant en tourment ${ }^{44}$.

Aussi, ma chere deesse, à vostre regard les autres ne me sont rien ${ }^{45}$.

Mon cœur, m'amour, mon tout, Mon cœur, m'amour, mon tout, vous vous moquerez de moy, \& dites que que ce n'est rien que mon tourment. A Dieu ne plaise, ma belle ame $e^{46}[\ldots .$.$] .$

A la plus aymée \& plus belle Bergere de l'univers, le plus infortuné \& plus fidelle de ses serviteurs envoye le salut que la fortune luy denie ${ }^{47}$.

Dans la péroraison, l'épistolier amplifie sa protestation d'amour exclusif et éternel au moyen des métaphores topiques du servage amoureux :

je m'asseure que cela vous sera agreable, pour gratifier vostre pauvre Floride, qui na jamais esté contente que par voz contentemens, comme celle qui aime sa vie que pource qu'elle est à vous ${ }^{48}$.

je continueray en mon obeissance, afin que si durant ma vie je n'ay peu vous asseurer de ma fidelité, les champs Elisées pour le moins, \& les ames bien-heureuses qui y sont, reconnoissent que je suis le plus fidelle, comme le plus infortuné de vos serviteurs ${ }^{49}$. 
Je m'estime le plus heureux berger du monde, puis que je suis à vous et ne treuve rien de si doux que vous faire service : belle Diane faites durer ce bonheur aussi longtemps que ma vie, et me nommez tousjours vostre, SILENE ${ }^{50}$.

Enfin, les lieux communs qui amplifient le thème de la lettre pourraient parfois sans difficulté être rangés dans les rubriques des anthologies mondaines. Exception notable, l'auteur anonyme de la Mariane du Filomène use des allégories typiques de ce que la postérité appellera le "style Nervèze $\mathrm{e}^{51}$ ", or ces tours particulièrement outranciers se présentent sous une forme bien plus brève dans les anthologies de la même époque :

Mon esperance est du tout morte, car je voy bien helas que ce fleuve de douceur, dont m'avez quasi de nectar et d'ambroisie abreuvé l'ame pour un temps, ne coule plus a present pour moy, plus seulement ne distille, la source en est jà tarie, si qu'altéré d'icelles languissant par trop, je resemble la fleur seiche par faute d'eau, \& bruslée à l'ardeur du Soleil ${ }^{52}$.

L'analogie principale de l'allégorie (l'espérance est un fleuve) est divisée en plusieurs sous-thèmes concrets (le nectar, la source, la fleur asséchée) qui construisent une fiction en miniature au sein de l'énoncé épistolaire, au risque de sa cohérence. Ce goût pour l'allégorie alambiquée, bien représenté dans les romans situés à la cour ou dans un espace urbain $^{53}$, est rare dans les anthologies mondaines et les fictions pastorales.

Dans les manuels mondains, au contraire, le goût de la formule frappante va de pair avec une abréviation de l'énoncé métaphorique. En témoigne cet exemple tiré de la rubrique « Absence » des Marguerites françoises :

Mon ame desja demy consommée dans ses propres flammes, vesve de sa puissance, souspire ses peines, qui prennent leur origine de vostre absence ${ }^{54}$.

Les métaphores du feu et des soupirs sont encadrées par les expressions littérales de l'«ame » et de l'«absence » qui contrôlent étroitement l'interprétation de l'énoncé. Les lettres enchâssées dans la fiction pastorale arborent elles aussi des marguerites maintenues dans les bornes d'une brève période :

le temps qui guerit les maux des autres, augmente tous les jours le mien, \& l'absence qui efface les images des sujets aymez, me peint la vostre à chaque moment de plus vives et plus sensibles couleurs ${ }^{55}$.

Demain sera le dernier jour de ma vie, si pour le moins on doit appeler mort ce qui ravit toute espece de contentement ${ }^{56}$.

L'épanouissement des fleurs du bien-dire dans les épîtres des bergers se présente comme la marque d'une écriture spontanément spirituelle, qui parvient à renfermer dans une formule remarquable les subtilités du sentiment amoureux. Resserrés sous leur forme la plus brillante dans ces brèves marguerites, les lieux communs sont aussi à l'œuvre dans les raisonnements des épistoliers, à une échelle cette fois macrotextuelle.

\section{Les raisons du cœur : casuistique épistolaire et psychologie romanesque}

32 Si l'imaginaire du parler roman réduit ce dernier à une collection d'hyperboles et de métaphores topiques, la pratique effective de ce dernier dépasse largement cette dimension de pure élocution. Il s'agit, à travers ce langage raffiné, de donner une représentation délicate des diverses passions de l'âme. Celle-ci trouve un terrain d'exercice privilégié dans le cadre des épîtres insérées. Comme dans les plaintes et dans les poèmes, les lettres offrent une modulation à la première personne du langage amoureux qui, dans la narration, prend la forme d'une représentation à la troisième 
personne. Mais l'expression épistolaire se rapproche de la voix narratoriale par son goût pour le raisonnement. La lettre enchâssée intervient toujours dans le cadre d'une situation problématique qu'elle a vocation à résoudre, par les moyens de la rhétorique judiciaire ou délibérative. L'énoncé épistolaire développe ainsi une casuistique amoureuse qui révèle les subtilités parfois sournoises du langage amoureux, et les feintes stratégiques de son apparent naturel. Dans ce travail du lieu commun, l'éloquence féminine n'est pas en reste. Bergères et bergers sont à égalité dans une tendance à rationaliser leur cause pour parvenir à leurs fins. Le discours épistolaire introduit ainsi dans la trame du récit des patrons d'analyse psychologique importés de la rhétorique et adaptés au goût du roman pour la complexification de l'intrigue amoureuse.

Dans le Paradis d'amour de Laudun d'Aigaliers, le héros Laudes, s'excusant auprès de Magdalaine de l'avoir trompée avec une nymphe, attire l'attention de sa destinataire sur l'éventuelle mauvaise foi qui sous-tend son raisonnement judiciaire :

Parquoy ma belle, soyez moy tant favorable et misericordieuse, que d'escouter quelques unes de mes excuses, et jaçoit qu'elles soient sophistiques, les prendre pour partie du payement, le reste estant remis à vostre accoustumée douceur ${ }^{57}$.

L'épistolier justifie ensuite son comportement en assurant qu'il n'a été infidèle qu'en rêve : en conséquence, la nymphe avec laquelle il a trompé son amante n'était autre que le fantasme de Magdalaine elle-même. Loin d'être infidèle, il est excessivement fidèle :

Helas! (ma chere pucelle) le grand soucy et soin, que j'ay tousjours eu de vous servir, est cause que dormant, mon esprit ne pouvoit reposer, ains se representoit fantastiquement, quelques especes intelligibles, comme celle de ceste Nymphe ${ }^{58}$.

Les lettres délibératives accusent des stratégies parfois retorses : les lettres de conseil recommandent souvent, avant tout, ce qui est dans l'intérêt de l'épistolier, et non du destinataire. Dans cette épître d'Astrée, celle-ci, tout en prétendant dissuader Céladon d'être son serviteur, revendique avec orgueil son tempérament tyrannique :

Qu'est-ce que vous entreprenez Celadon? en quelle confusion vous allez-vous mettre? croyez moy qui vous conseille en amye, laissez ce dessein de me servir, il est trop plein d'incommoditez: quel contentement y esperez-vous? je suis tant insupportable que ce n'est guere moins entreprendre que l'impossible: il faudra servir, souffrir, et n'avoir des yeux, ny de l'Amour que pour moy: car ne croyez point que je veuille avoir à partir avec quelqu'autre, ny que je reçoive une volonté à moitié mienne : je suis soupçonneuse, je suis jalouse, je suis difficile à gagner, et facile à perdre ; et puis aisée à offenser, et très mal-aisée à rappaiser ; la moindre doute est en moy une asseurance ; il faut que mes volontés soient des destinées, mes opinions des raisons, et mes commandements des lois inviolables. Croyez moy, encore un coup; retirez-vous, Berger, de ce dangereux labyrinthe, et fuyez un dessein si ruïneux. Je me recognois mieux que vous, ne vous figurez de pouvoir à la fin changer mon naturel, je rompray plustost que de plier, et ne vous plaignez à l'avenir de moy, si à ceste heure vous ne croyez ce que je vous en dis ${ }^{59}$.

L'argumentation de la bergère repose sur une inversion de la métaphore topique du servage amoureux. Alors qu'il fait d'habitude l'objet d'une protestation masculine et qu'il s'accompagne d'un éloge de la maîtresse aimée, il est ici énoncé par une femme qui recommande (prétendument) l'émancipation, et présente sa propre tyrannie comme un vice. Mais cette affectation d'auto-dénigrement vise en réalité à asseoir une domination plus absolue, puisqu'Astrée, en reconnaissant avec franchise les défauts de son « naturel », s'arroge également le droit de ne jamais avoir à se corriger. 
Le tempérament raisonneur des épistoliers tend ainsi à complexifier l'éthique amoureuse des bergeries, à travers d'infinies négociations de couples qui aboutissent plus souvent à une noyade qu'à un compromis ${ }^{60}$. Poussée jusqu'à l'extrême, la subtilité casuistique s'apparente au jeu galant, comme dans ce raisonnement de la Diane de Montemayor, où la bergère éponyme, accusée de ne pas aimer son berger, renvoie l'accusation à son envoyeur :

Tu me dis, que je ne t'ayme point tant comme je le doibs : je ne sçay à quoy tu le cognois, ny comment je te puisse aymer davantage. Regarde que maintenant il n'est plus temps de ne me point croire, puis que tu vois que la grande amour que je te porte, me force à croire ce que tu me dis de ton affection. Souventes-fois j'imagine qu'ainsi comme tu penses que je ne t'ayme point, t'aymant plus que moy mesme, aussi peux tu penser que tu m'aymes, m'ayant en hayne et horreur ${ }^{61}$.

Les enthymèmes à la chaîne de la bergère pourraient être reformulés de la manière suivante : si tu m'aimes, alors tu dois croire en mes serments d'amour, et si tu ne crois pas en mes serments d'amour, alors tes serments d'amour sont mensongers.

La visée modélisatrice de tels raisonnements paradoxaux nous semble être confirmée par la présence, d'un texte pastoral à l'autre, des mêmes lieux communs légèrement remaniés en fonction de la situation narrative dans laquelle ils s'insèrent. Ainsi, Astrée reprend le cas litigieux de Diane, mais lui apporte une solution différente :

Vous ne voulez pas croire que je vous ayme, et desirez que je croye que vous m'aimez: si je ne vous aime point, que vous profitera la creance que j'auray de vostre affection? [...] je ne veux pas seulement que vous sçachiez que je croy que vous m'aymez; mais je veux de plus, que vous soyez asseuré que je vous ayme ${ }^{62}[. .$.$] .$

L'enthymème repose ici non pas sur la réciprocité de la confiance mais sur celle de la volonté : si vous voulez que je croie à votre amour, alors, réciproquement, j'ai le droit d'exiger que vous croyiez au mien.

Le trésor épistolaire de la fiction pastorale tend ainsi à constituer une encyclopédie des lieux communs amoureux, épuisant tous les cas possibles et imaginables avec une fantaisie qui excède largement la vertu modélisatrice pour devenir un pur jeu de langage et d'esprit.

\section{Conclusion}

L'enchâssement épistolaire dans la fiction pastorale répond à un goût d'époque pour la lettre amoureuse, et confirme la vocation du roman à constituer une encyclopédie rhétorique mondaine, offrant au public un apprentissage accéléré et ludique des formes du bien-dire.

L'analyse rhétorique de l'épître insérée dans la bergerie fait apparaître plusieurs spécificités du genre : la lettre est un ornement rare de ce type de fiction, sans doute parce qu'elle est perçue comme une pièce maîtresse des pratiques mondaines et qu'elle ne peut sans invraisemblance envahir l'imaginaire arcadien. Elle partage cependant bien des traits avec les lieux communs des manuels de rhétorique mondaine, et il serait intéressant d'étudier l'éventuelle circulation des marguerites dans les recueils et les fictions en prose. La lettre insérée développe enfin une tendance au raisonnement et à la casuistique amoureuse qui participe à la spécialisation du roman de cette époque dans l'analyse psychologique. 
41 Si le naturel des épîtres de fictions pastorales est bien une mythologie, dont témoigne l'orchestration pathétique de sa disposition, le brillant de son élocution et la subtilité de ses raisonnements, il reste que cette mythologie, pleinement intégrée au dispositif rhétorique qui sous-tend la textualité romanesque de l'époque renaissante et baroque, participe à la constitution d'une poétique romanesque moderne. Elle nourrit, en effet, la dynamique métalangagière du texte fictionnel par laquelle l'événement narratif rebondit principalement sur des actes de langage et des manières de dire amoureux. Par cette disposition anthologique, la fiction en prose s'inscrit dans l'imaginaire langagier de la conversation à bâtons rompus et trouve dans l'épître une modulation essentielle, puisque la rhétorique épistolaire constitue une pièce maîtresse de cet imaginaire.

Si l'on compare maintenant les cinq monodies des Lettres portugaises aux brèves pièces de nos bergers, force est de constater qu'on assiste, dans les années 1660, à une profonde modification des pratiques. Le désordre, la longueur, le style sublime des lettres de la religieuse, s'ils rappellent à bien des égards les procédés de la plainte enchâssée dans les romans baroques, diffèrent en revanche en tous points de la brièveté spirituelle et raisonneuse des épîtres pastorales, qu'on pourrait reclasser, en suivant a posteriori la terminologie de Madeleine de Scudéry, en « lettre galante » et non plus amoureuse ${ }^{63}$.

\section{NOTES}

1. Ch. Sorel, «Censure des fables et des romans ", De la connoissance des bons livres, ou examen de plusieurs autheurs, Paris, A. Pralard, 1671, p. 101-102.

2. Ibid., p. 102.

3. Voir M. Fumaroli, «Académie, Arcadie, Parnasse ", L'École du silence. Le sentiment des images au XVII ${ }^{e}$ siècle, Paris, Flammarion, 1998, p. 32-48.

4. Voir A. Petit, Le Discours romanesque des passions. Rhétorique et poétique des passions au XVII siècle, thèse de doctorat dir. par Delphine Denis, soutenue à l'université Paris-Sorbonne en oct. 2016, p. 320.

5. Voir D. Denis, «Le roman, genre polygraphique?» dans Littératures classiques, $\mathrm{n}^{\circ} 49,2003$, p. 339-370, C. Esmein, L'Essor du roman, Discours théorique et constitution d'un genre au XVII siècle, Paris, Honoré Champion, 2008, p. 404 et M.-G. Lallemand, Les Longs romans du XVII siècle. Urfé, Desmarets, Gomberville, La Calprenède, Scudéry, Paris, Garnier, 2013, p. 295-303.

6. Voir V. Benhaïm, "Les Thresors d'Amadys ", dans Nicole Cazauran dir., Les Amadis au XVI siècle en France, Paris, Éditions de la rue d'Ulm, 2000, p. 157-181.

7. Voir S. Duval, « De la marguerite aux pièces agréables : les choix de belles proses à l'âge baroque ", dans C. Bohnert et Fr. Gevrey dir., L'Anthologie. Histoire et enjeux d'une forme éditoriale du Moyen Âge au XXI siècle, Reims, Éditions et presses universitaires de Reims, 2014, p. 249-268.

8. Comme l'atteste le titre de F. de Belleforest: La Pyrenée, et pastorale amoureuse, contennant divers accidens amoureux, descriptions de paysages, histoires, fables et occurrences des choses, advenues de nostre temps, servant comme l'avant-coureur de l'Adolescence: divisée en deux livres, Paris, G. Mallot, 1571. 
9. Sur ces dispositifs éditoriaux, voir H.-J. Martin, La Naissance du livre moderne (XVI'-XVII ${ }^{e}$ siècle), Paris, Éditions du cercle de la librairie, 1999, p. 326-327.

10. La parution du recueil de Puget de La Serre en 1619 (Le Bouquet des plus belles fleurs de l'éloquence de ce temps), puis du recueil Faret en 1627 (voir É. Méchoulan et M. Bombart, Politiques de l'épistolaire au XVII siècle. Autour du Recueil Faret, Paris, Garnier, 2011) témoignent de cette montée en puissance de l'anthologie épistolaire, de même que le retentissement des premières Lettres de Guez de Balzac en 1624, où figurent plusieurs épîtres amoureuses (voir M. Bombart, Guez de Balzac et la querelle des "Lettres" : écriture, polémique et critique dans la France du premier XVII siècle, Paris, H. Champion, 2007). Sur cet essor éditorial, voir R. Zuber, Les Belles infidèles et la formation du goût classique, Paris, A. Colin, 1968, p. 41 et M. Fumaroli, L'Âge de l'éloquence. Rhétorique et « res literaria » de la Renaissance au seuil de l'époque classique [1980], Genève, Droz, 2002, p. 266.

11. Voir M. Fumaroli, «De l'âge de l'éloquence à l'âge de la conversation: la conversion de la rhétorique dans la France du XVII ${ }^{\mathrm{e}}$ siècle », dans B. Bray et Chr. Strosetski dir., Art de la lettre, art de la conversation à l'époque classique en France, Paris, Klincksieck, 1995, p. 25-44.

12. É. Pasquier, "Epistres", Recueil des rymes et prose de E.P., Paris, Vincent Sertenas, 1555, p. 30-50. Voir Bernard Bray, «Les "lettres amoureuses" d'Etienne Pasquier, premier roman épistolaire français ? ", Cahiers de l'Association Internationale des études françaises (CAIEF), 1977, $\mathrm{n}^{\circ}$ 29, p. 133-145.

13. Cette mode est notamment marquée par l'ouvrage de P. de Deimier (Lettres amoureuses, non moins pleines de belles conceptions que de beaux desirs. Ensemble la traduction de toutes les Epistres d'Ovide, Paris, G. Sevestre, 1612 et le recueil collectif de N. Renouard (Les Fleurs de l'éloquence françoise extraictes des épistres hérö̈ques d'Ovide, Paris, A. Estoc, 1615). Voir M.-Cl. Chatelain, Ovide savant, Ovide galant : Ovide en France dans la seconde moitié du XVII siècle, Paris, H. Champion, 2008.

14. Voir B. Bray, L'Art de la lettre amoureuse. Des manuels aux romans (1550-1700), La Haye/Paris, Mouton, 1967, en particulier p. 27-28.

15. Érasme, De conscribendis epistolis [1522], Lugduni, T. Paganum, 1557, p. 269-272.

16. P. Fabri, Le Grand et Vrai Art de pleine rhétorique [1521], éd. A. Héron, Imprimerie Espérance Cagniard, 1889, p. 197.

17. Ibid., p. 227.

18. Ibid., p. 228.

19. Sur le classement pragmatique des différentes espèces de lettre dans les traités d'épistolographie, voir C.Lignereux, «L'art épistolaire de l'âge classique comme champ d'application du savoir rhétorique », Exercices de rhétorique [En ligne], 6|2016, URL: http:// journals.openedition.org/rhetorique/441.

20. P. Fabri, Le Grand et Vrai Art, op. cit., p. 228.

21. Voir l'édition numérique de l'Instruction par C. Lignereux : Exercices de rhétorique [En ligne], 6 | 2016. Voici quelques exemples des épîtres amoureuses qui sont données à la suite de cette Instruction: "Lettres d'amour sur toutes sortes de sujets, \& premierement de presentation de service », p. 180-183 ; "Lettres sur l'absence», p. 191-195; «Lettres pour se plaindre d'une inconstance ", p. 205-207 (J. Puget de La Serre, Le Secrétaire à la mode, Rouen, R. Doré, 1651). On notera aussi l'exemple notable (et signalé par C. Lignereux dans le même article) de P. Jacob qui mentionne la « lettre d'amour » dans sa rubrique des lettres délibératives : Le Parfait secrétaire, ou la Manière d'escrire et de respondre à toute sorte de lettres par Preceptes et par Exemples, Paris, A. de Sommaville, 1646. À l'époque classique, en revanche, la lettre d'amour est bien représentée dans les théories épistolographiques.

22. Voir A. Petit, Le Discours romanesque des passions. Rhétorique et poétique des passions au XVII siècle, thèse citée, p. 320-324.

23. Sur ces topoï du roman d'amour, voir Fr. Greiner, Les amours romanesques de la fin des guerres de religion au temps de L'Astrée (1585-1628), Paris, H. Champion, 2008, notamment p. 151-187.

24. Voir G. S. Du Verdier, La Diane Françoise, Paris, A. de Sommaville, 1624, p. 405-406. 
25. Voir P. de Laudun d'Aigaliers, Le Paradis d'amour, ou la chaste matinée du fidell'amant, Rouen, 1606, C. Le Villain, $\mathrm{f}^{\circ} 44, \mathrm{r}^{\mathrm{o}}-\mathrm{v}^{\mathrm{o}}$.

26. Voir G. S. Du Verdier, La Diane Françoise, op. cit., p. 168-169, et C.-Fr. O. de Préfontaine, La Diane des Bois, Rouen, J. Caillove, 1632, p. 82.

27. Voir P. de Laudun D'Aigaliers, Le Paradis d'amour, op. cit., fo $51, \mathrm{v}^{\circ}$.

28. Ch. Sorel, Le Berger extravagant, Paris, T. du Bray, 1627, IV, p. 277.

29. Ces apostrophes et ces exclamations sont, comme l'a montré A. Petit, des formes caractéristiques du narrateur «intéressé » du roman d'amour antérieur aux années 1620 : Le Discours romanesque des passions, op. cit., p. 190-194.

30. N. de Montreux, Le Premier Livre des bergeries de Juliette, de l'invention d'ollenix du Mont-Sacré, Lyon, J. Veyrat, 1592, I, III, fo 90, $\mathrm{v}^{\circ}$.

31. H. d'Urfé, L'Astrée. Première partie, op. cit., I, p. 141.

32. C.-Fr. O. de Préfontaine, La Diane des Bois, op.cit., p.82-83 (le texte écrit bien «triste souspirs »).

33. H. d'Urfé, L'Astrée. Deuxième partie, op. cit., III, p. 146.

34. Voir S. Grosse, Les Manuels épistolographiques français entre traditions et normes, Paris, H. Champion, 2017, p. 295-296.

35. Ch. Sorel, "De la manière de bien écrire ", De la connoissance des bons livres, ou examen de plusieurs autheurs, Paris, A. Pralard, 1671, p. 296.

36. Ibid., p. 298.

37. Un tel procédé, en accentuant l'exemplarité du cas d'amour traité dans l'épître, rapproche la rhétorique épistolaire de celle de l'histoire insérée, autre pièce caractéristique de la polygraphie romanesque qui est elle aussi fréquemment inaugurée par de telles sentences.

38. H. d'Urfé, L'Astrée. Première partie [1612], éd. D. Denis et alii, Paris, H. Champion, 2011, VI, p. 371. Dans toutes les citations de la présente étude, c'est moi qui souligne en gras.

39. H. d'Urfé, L'Astrée. Première partie, op. cit., p. 371.

40. Ibid.

41. Ibid.

42. C. Esmein, «"Parler roman": Imaginaire de la langue et traits de style romanesques au XVII siècle ", Revue d'Histoire littéraire de la France (RHLF), CIX, 2009, p. 85-99.

43. Sur la place des bergères dans les formes insérées chez Urfé, voir M.-G. Lallemand, «Éloquence masculine et éloquence féminine dans L'Astrée d'Honoré d'Urfé », dans A. Paupert et Ch. Liaroutzos dir., La Discorde des deux langages. Représentations des discours masculins et féminins du Moyen Âge à l'Âge classique, Textuel, $\mathrm{n}^{\circ}$ 49, 2006, p. 171-185.

44. P. de Laudun D'Aigaliers, Le Paradis d'amour, op. cit., fo $51, \mathrm{v}^{\circ}$.

45. Ibid.

46. Ibid.

47. H. d'Urfé, L'Astrée. Deuxième partie [1614], éd. D. Denis et alii, Paris, H. Champion, 2016, III, p. 146.

48. Fr. Béroalde de Verville, Les Aventures de Floride, Tours, J. Mettayer, 1594, II, 1, 13, fo 45, $\mathrm{r}^{\circ}$.

49. H. d'Urfé, L'Astrée. Deuxième partie, op. cit., III, p. 146.

50. G. S. Du Verdier, La Diane françoise, op. cit., p. 29.

51. Voir R. Zuber «Grandeur et misère du style Nervèze », Les émerveillements de la raison, Paris, Klincksieck, 1997, p. 83-98.

52. Anonyme, La Mariane du Filomène, Paris, C. de Montr'oeil et G. Richer, 1596, p. 198.

53. Voir S. Duval, La Prose poétique du roman baroque (1571-1670), Paris, Garnier, 2017, p. 440-444.

54. Fr. Desrues, Les Marguerites françoises, op. cit., p. 4.

55. C.-Fr. O. de Préfontaine, La Diane des Bois, op. cit., p. 82.

56. H. d'Urfé, L'Astrée. Deuxième partie, op. cit., IV, p. 224. 
57. P. de Laudun d'Aigaliers, Le Paradis d'amour, op. cit., fo $45, \mathrm{r}^{\circ}$.

58. Ibid.

59. H. d'Urfé, L'Astrée. Première partie, op. cit., III, p. 206.

60. Cette complexification de l'intrigue par le biais de l'épistolaire prend également la forme des multiples falsification, lectures indiscrètes et vols de lettres auxquels se livrent les bergers. M.G. Lallemand, à partir d'une étude des romans de Scudéry a montré l'existence de cet «épistolaire romanesque » dans les fictions pastorales du début du XVII ${ }^{\mathrm{e}}$ siècle : La Lettre dans le récit. Étude de l'œuvre de $M^{\text {elle }}$ de Scudéry, Tübingen, Gunter Narr Verlag, 2000, p. 145 et sq.

61. N. Colin (trad.), Les Sept Livres de la Diane de Montemayor, traduits d'espagnol en français, Reims, J. de Foigny, 1578, fo $5 \mathrm{v}^{\mathrm{o}}-\mathrm{f}^{\mathrm{o}} 6 \mathrm{r}^{\mathrm{o}}$.

62. Ibid., I, 3, p. 207.

63. Cette distinction entre la lettre d'amour naturelle et la lettre galante spirituelle est énoncée dans la célèbre conversation sur les lettres de la Clélie: M. de Scudéry, Clélie. Histoire romaine. Quatrième partie [1658], éd. Ch. Morlet-Chantalat, Paris, H. Champion, 2004, p. 417. Voir l'édition et l'analyse qu'en donne D. Denis : «De L'Air galant» et autres conversations (1653-1684). Pour une étude de l'archive galante, Paris, H. Champion, 1998, p. 139-158.

\section{AUTEUR}

\section{SUZANNE DUVAL}

Université de Lausanne 